\title{
Macrophage profile in primary versus secondary liver tumors
}

\author{
Elena-Roxana Avădănei ${ }^{*}$, Piotr M. Wierzbicki ${ }^{*}$, Simona-Eliza Giuşcă ${ }^{3}$, \\ Adriana Grigorass ${ }^{1}$, Cornelia Amălinei ${ }^{1}$, Irina-Draga Căruntu ${ }^{1}$
}

\author{
${ }^{1}$ Department of Morphofunctional Sciences - Histology, "Grigore T. Popa” University of Medicine \\ and Pharmacy, Iaşi, Romania \\ ${ }^{2}$ Department of Histology, Medical University of Gdansk, Poland \\ ${ }^{3}$ Department of Pathology, "Sf. Spiridon” University Hospital, Iaşi, Romania
}

\begin{abstract}
Macrophages are important components of the tumor-associated infiltrate and are qualified as one of the major players of the cancer-related inflammation. It was shown that tumor cells can either stimulate or mediate apoptosis of tumor-associated macrophages (TAMs). To date, there is no general agreement regarding the influence of TAMs and their numbers on the progression of hepatocellular carcinoma (HCC) and hepatic metastases (HM). To analyze the presence of TAMs and compare their numbers in intratumoral (IT) and peritumoral (PT) areas with the clinical outcome of HCC and HM patients. Biopsies from $35 \mathrm{HCC}$ and $39 \mathrm{HM}$ cases were analyzed. Clinical and follow-up data was enrolled for each case; the colorectal cancer was the origin of $26 \mathrm{HM}$ patients. TAMs were identified by immunohistochemistry using anti-CD68 monoclonal antibody. The quantitative assessment was performed by determining the mean number of CD68-positive cells in IT and PT areas in HCC and HM. Two threshold methods were applied: threshold 1 (T1) was calculated with the use of (-log) Cox method; threshold 2 (T2) was considered as 1/3 TAMs number of group's mean. For statistical analyses Mann-Whitney U-test, Spearman's correlation, Cox proportional hazard and Kaplan-Meier tests were applied. To date, $36.12 \% \mathrm{HCC}$ and $27.78 \% \mathrm{HM}$ patients were alive, median survival was 5 and 17 months for HCC and HM, respectively $(P=0.05)$. We found significant two-fold decrease of TAMs numbers between IT $v s$. PT territories in both HCC and HM. A positive correlation between numbers of PT and IT TAMs was observed in HM group $\left(\mathrm{r}_{\mathrm{s}}=0.48, P<0.05\right)$ but not in HCC. The number of TAMs was not associated with any studied clinical factor. Univariate Cox regression analysis showed that tumor stage $\leq$ II $(P=0.01)$ and increased number of PT TAMs $(P=0.06$, only when T2 value was applied) were associated with favorable prognosis in HCC ( $\mathrm{HR}=2.614$ and 2.457, respectively). Univariate and multivariate Cox analyses in HM revealed favorable prognosis for histological grade $\leq \mathrm{G} 2$ and one lobe tumors $(P=0.021$ and $0.045 ; \mathrm{HR}=0.395$ and 0.438 , respectively $)$. Survival analysis retained the impact of increased TAMs numbers in peritumoral areas $(P=0.03)$, tumor stages in HCC $(P=0.007)$, lobes' number $(P=0.007)$ and histological grade $(P=0.005)$ on HM patients' outcome. In HCC and HM the low number of TAMs in intratumoral areas was related to the tumor cell microenvironment. The increased peritumoral TAMs number in primary liver tumors was associated with better prognosis. (Folia Histochemica et Cytobiologica 2014, Vol. 52, No. 2, 112-123)
\end{abstract}

Key words: macrophages; CD68; hepatocellular carcinoma; liver metastases; quantitative analysis; peritumoral; intratumoral; prognosis

Correspondence address: I.-D. Căruntu, M.D., Ph.D.

Department of Morphofunctional Sciences - Histology,

"Grigore T. Popa" University of Medicine and Pharmacy, Iaşi

16 University St., Iaşi, 700115 Romania;

tel.: +40727003700

e-mail: irinadragacaruntu@gmail.com

*These authors equally contributed to this paper 


\section{Introduction}

The hepatic tumor microenvironment expresses a unique cellular and molecular profile which may be characterized by cellular and non-cellular components [1] with interdependent functions and reciprocal signaling during their involvement in the process of carcinogenesis [2]. Macrophages, dendritic cells, mast cells, cytotoxic natural killer cells, and $\mathrm{T}$ cells of the hepatic microenvironment are responsible for the development of innate immune responses adapted to a tolerant immunological status $[1,3,4]$ which is regarded as procarcinogenic. Moreover, liver extracellular matrix (ECM) contains a large diversity of molecules secreted by both parenchymal and non-parenchymal liver cells, such as growth factors, proteolytic enzymes with their specific inhibitors, and inflammatory cytokines [1,2] which affect function of both immune and non-immune cells.

Macrophages are important components of the tumor-associated infiltration, being considered as major players of the cancer-related inflammation [5]. Tumor-associated macrophage (TAM) population is heterogeneous with two major subtypes described: classically activated (type I or M1) and alternatively activated macrophages (type II or M2) [6-8]. Referring the origin of the TAMs, it was hypothesized that microenvironmental factors induce the differentiation toward one specific phenotype [8] since supplementary and intermediary phenotypes were noticed by some studies [9]. Macrophages' heterogeneity, based on their functional plasticity [10], resulted in the comparison of the pro- or anti-tumor activity of TAMs to a "double-edges sword" [7, 11] or "Janus face" [12].

Recent studies sustain that TAMs represent an unfavorable prognostic factor in hepatocellular carcinoma (HCC), since the TAM-secreted molecules stimulate the growth and survival of HCC cells, the epithelial-mesenchymal transition (EMT), tumoral angiogenesis, ECM remodeling, and suppression of the anti-tumoral immunity [13, 14]. On the other hand, TAMs association to an improved postoperative survival and their role as positive prognostic factor in HCC were supported by other research groups $[15,16]$. In the studies of TAMs involvement in the development of hepatic metastases (HM), the most numerous were related to the colorectal cancer (CRC)-associated metastases [17-21]. Similarly to HCC, macrophages in $\mathrm{HM}$ can act against metastatic cells in a direct cytotoxic way or by secretion of TNF and proteases [22], however, TAMs can also support survival and differentiation of malignant cells via secretion of growth factors [23] or metalloproteinases [24].

Within this context, the objective of our study was a qualitative and quantitative characterization of TAMs in HCC vs. HM in intra- and peritumoral areas, followed by statistical assessment of TAMs as a possible prognostic factor.

\section{Material and methods}

Patients. The retrospective study was approved by the Ethics Committee of the "Grigore T. Popa" University of Medicine and Pharmacy, Iaşi, Romania, based on the patients' informed written consent for the usage of the biologic material for research. The study included 74 patients diagnosed with hepatocellular carcinoma (HCC, 35 cases - Group 1) and hepatic metastases of different origins (HM, 39 cases - Group 2) between January 2009 and December 2011, at the "Sf. Spiridon" University Hospital, Iaşi, Romania. The patients were surgically treated, without preoperative chemotherapy or radiotherapy. Within HM group the origin of the hepatic metastases was: colorectal cancer -24 cases ( 18 colon cancer, 6 rectal cancer), stomach -8 cases, pancreatic -2 cases, gallbladder -1 case, duodenal -1 case, ovarian -1 case, breast -1 case, adrenal gland cancer -1 case. The histopathological examinations were performed in the hospital's pathology laboratory, where the harvested tumor fragments were processed into paraffin blocks using classic histological technique. The patients' clinicopathological characteristics were summarized in Table 1 and Table 2. The median survival rates were 5 months for HCC and 17 months for HM cases. On $31^{\text {st }}$ of January $201336.12 \%$ of HCC patients and $27.78 \%$ of HM patients were alive. All recorded patients' deaths were associated with diagnosed cancer disease.

Immunohistochemistry. For immunohistochemical (IHC) study formalin fixed, paraffin-embedded (FFPE) $4 \mu \mathrm{m}$-thick tissue sections, corresponding to the areas previously examined by pathologist, were displayed on Superfrost Plus (Thermo Fischer Scientific, Fitchburg, WI, USA) slides. Sections were deparaffinized in two xylene baths $1^{\text {st }}$ at $58^{\circ} \mathrm{C}$ for $30 \mathrm{~min}$ and $2^{\text {nd }}$ at room temperature (RT) for $10 \mathrm{~min}$. The rehydration was made in four following alcohol solutions $(100 \%, 90 \%, 80 \%$, and $70 \% \mathrm{EtOH})$ for $10 \mathrm{~min}$ each. For antigen retrieval, we used heat-induced epitope retrieval (HIER), by immersing the slides in a citrate buffer ( $\mathrm{pH}$ 6.0) (Leica Biosystem, Newcastle Ltd, United Kingdom) at $98^{\circ} \mathrm{C}$ for $30 \mathrm{~min}$. After cooling the slides at room temperature (RT) for $20 \mathrm{~min}$ the endogenous peroxidase was blocked by incubation with $3 \%$ hydrogen peroxide solution (RT, for 10 $\mathrm{min}$ ), followed by one wash with distilled water for $5 \mathrm{~min}$. For the IHC identification of macrophages, slides were incubated with the primary antibody (1:1200, mouse anti-human CD68 monoclonal antibody, code: NCL-CD68-KP1, Novocastra, Leica Biosystem), overnight at $4^{\circ} \mathrm{C}$. The immunoreaction was developed with Novolink polymer kit (Novocastra, Leica Biosystem) as following: incubation with biotinylated secondary antibody and with the enzyme, 
Table 1. Clinicopathological characteristics of patients — Group 1, hepatocellular carcinoma (HCC)

\begin{tabular}{|c|c|c|c|}
\hline Variables & $\begin{array}{c}\text { Variable } \\
\text { description }\end{array}$ & $\begin{array}{c}\text { Cases } \\
\text { number }\end{array}$ & $(\%)$ \\
\hline Age & $\begin{array}{l}\text { Mean } \pm \mathrm{SD} \\
\text { median, } \\
\text { range (years) }\end{array}$ & $\begin{array}{c}62.9 \pm 10.7 \\
64,23-83\end{array}$ & \\
\hline$\leq 63$ & & 17 & 48.57 \\
\hline$>63$ & & 18 & 51.42 \\
\hline \multicolumn{4}{|l|}{ Gender } \\
\hline Female & & 12 & 34.28 \\
\hline Male & & 23 & 65.71 \\
\hline \multicolumn{4}{|l|}{ Tumor stage, } \\
\hline Stage I & T1N0M0 & 5 & 14.28 \\
\hline Stage II & T2N0M0 & 13 & 37.14 \\
\hline Stage IIIa & T3N0M0 & 16 & 45.17 \\
\hline Stage IV & TxNxM1 & 1 & 2.8 \\
\hline \multicolumn{4}{|l|}{ Histological grade ${ }^{*, * *}$} \\
\hline G1 & $\begin{array}{c}\text { Well } \\
\text { differentiated }\end{array}$ & 13 & 37.14 \\
\hline G2 & $\begin{array}{l}\text { Moderately } \\
\text { differentiated }\end{array}$ & 15 & 42.86 \\
\hline G3 & $\begin{array}{c}\text { Poorly } \\
\text { differentiated }\end{array}$ & 7 & 20.00 \\
\hline
\end{tabular}

*pTNM and histological divisions according to [43]; **pTNM stages (IIIb, IIIc) and G4 were not diagnosed, therefore they were omitted

respectively, for $30 \mathrm{~min}$ each, at RT. Between each step of incubation (primary and secondary antibodies, enzyme), slides were washed in 3 baths of phosphate-buffer saline for 5 min each. For visualization, we used 3.3'-diaminobenzidine dihydrochloride (DAB, Novocastra, Leica Biosystem) for $3 \mathrm{~min}$ - the immunopositivity was confirmed through the presence of a brown product. The nuclei were then counterstained with hematoxylin and mounted in non-aqueous mounting medium. The negative control was made concomitantly by omitting the incubation with the primary antibody, and respecting all the other steps of the protocol. The positive control was made during the same run by using a tonsil tissue section.

Quantitative analysis. The quantitative assessment was performed, for each case, by counting the number of CD68-positive cells in 10 microscopic fields with the highest density of macrophages, at $200 \times$ magnification, separately for the intratumoral and peritumoral territories. Our method represented a modified variant of the semi-quantitative evaluation of macrophages proposed by Forssell and co-workers, in colon cancer [25]. The IHC evaluation was done independently by two histopathologists (E.R.A. and S.E.G.) trained in IHC interpretation, unaware of the clinical outcome. On the basis of the mean number of TAMs of each case, we have determined the mean number of macrophages for each studied group, separately for intratumoral (IT) and peritumoral (PT) territories.
Table 2. Clinicopathological characteristics of patients - Group 2, hepatic metastases (HM)

\begin{tabular}{|c|c|c|c|}
\hline Variables & $\begin{array}{c}\text { Variable } \\
\text { description }\end{array}$ & $\begin{array}{c}\text { Cases } \\
\text { number }\end{array}$ & $(\%)$ \\
\hline $\begin{array}{l}\leq 68 \\
>68\end{array}$ & $\begin{array}{l}\text { Mean } \pm \mathrm{SD} \\
\text { median, } \\
\text { range (years) }\end{array}$ & $\begin{array}{c}67.6 \pm 11.9 \\
70,35-86 \\
17 \\
22\end{array}$ & $\begin{array}{l}43.58 \\
56.41\end{array}$ \\
\hline $\begin{array}{l}\text { Gender } \\
\text { Female } \\
\text { Male }\end{array}$ & & $\begin{array}{l}18 \\
21\end{array}$ & $\begin{array}{l}46.15 \\
53.84\end{array}$ \\
\hline $\begin{array}{l}\text { Tumor stage } \\
\text { Stage IV }\end{array}$ & TxNxM1 & 39 & 100 \\
\hline $\begin{array}{l}\text { Histological grade } \\
\quad \text { G1 }\end{array}$ & $\begin{array}{c}\text { Well } \\
\text { differentiated }\end{array}$ & 3 & 7.69 \\
\hline $\mathrm{G} 2$ & $\begin{array}{c}\text { Moderately } \\
\text { differentiated }\end{array}$ & 20 & 51.28 \\
\hline G3 & $\begin{array}{c}\text { Poorly } \\
\text { differentiated }\end{array}$ & 14 & 35.89 \\
\hline G4 & Undifferentiated & 2 & 5.12 \\
\hline $\begin{array}{l}\text { Tumoral extension } \\
\text { One lobe } \\
\text { Many lobes }\end{array}$ & & $\begin{array}{l}26 \\
13\end{array}$ & $\begin{array}{l}66.66 \\
33.33\end{array}$ \\
\hline
\end{tabular}

Statistical analysis. Statistical analysis has been performed using MedCalc software (MedCalc Software, Ostend, Belgium) and GraphPad Prism ver. 6 (GraphPad Software Inc., La Jolla, CA, USA). Patients' characteristics were given in absolute and relative numbers or as mean values with standard error of the mean (SEM). Mean macrophage scores for each subgroup were further checked with the use of D'Agostino normality test. Mann-Whitney U test was used for statistical comparison between two variables whereas Kruskal-Wallis ANOVA test was utilized when more than two independent variables were analyzed. For checking the associations between variables, Spearman's correlation was utilized.

Estimation of thresholds. Two thresholds were estimated: research and analytical. The first threshold (T1) was calculated for TAMs using univariate Cox regressions analyses (-log test). Therefore, cases with lower mean score than T1 for the respective division were considered as subgroup 1 (or $\sqrt{ }$ ) whereas cases with higher mean macrophages score were treated as subgroup 2 (or $\uparrow$ ). The second threshold (T2) has been additionally used in order to verify if a lower value compared to the average value of the group might offer a superior refinement of the correlation with patients' survival. T2 was calculated as $1 / 3$ of macrophages mean number for each group and territory divisions. Thus, specimens with lower macrophage number than T2 were marked to subgroup 3 (or $\downarrow$ ) whereas cases with higher number than 


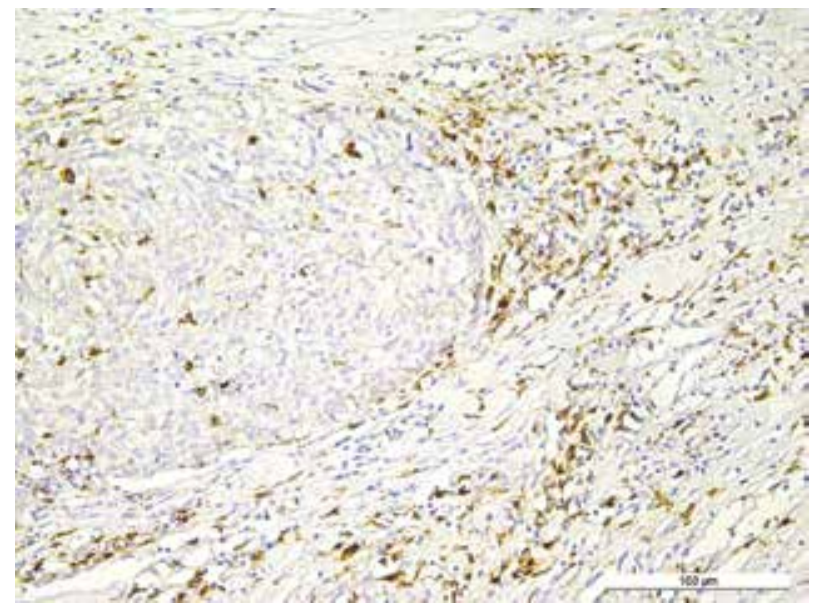

Figure 1. Pattern of macrophages distribution in HCC: numerous at the border of a tumoral nodule, very few dispersed within tumor (immunohistochemical, IHC, detection by anti-CD68 monoclonal antibody). Objective $\times 20$

T2 were scored to subgroup 4 (or $\boldsymbol{\uparrow}$ ). Both T1 and T2 were further considered in survival analyses. Fisher $2 \times 2$ test was utilized for the statistical comparison between subgroups based on T1/T2 classification.

Survival analysis. The Cox-Mantel proportional hazard regression model was used to evaluate the effect of explorative variables on survival of HCC and HM patients. First, univariate Cox regression analysis for every single variable was performed. Secondly, variables with a $P$ value $<0.05$ were included into multivariate Cox regression analysis with a variable selection via backward elimination. All associations were presented as hazard ratios (HR) with their 95\% confidence interval $(\mathrm{CI})$ and $P$ values. Kaplan-Meier estimations were performed to describe survival rates.

\section{Results}

\section{Qualitative evaluation}

Microscopic examination has revealed both in Group 1 - HCC and in Group 2 - HM, CD68 immunoreactive $\left(\mathrm{CD}^{+} 8^{+}\right)$dominant population in the periphery of tumoral islands or nodules, at the interface with the normal liver parenchyma (Figures $1-4$ ). This territory could be identified in all cases, being considered as the tumor's invasion front. Macrophages were identified as either single isolated cells or in small compact groups, inside the tumor, being mainly disposed in the area corresponding to the most external 2-3 cell layers (Figures 1-4). The qualitative evaluation, based on a visual, subjective appreciation of macrophages' presence did not reveal differences between the distribution (location) patterns of these cells in the HCC vs. HM.

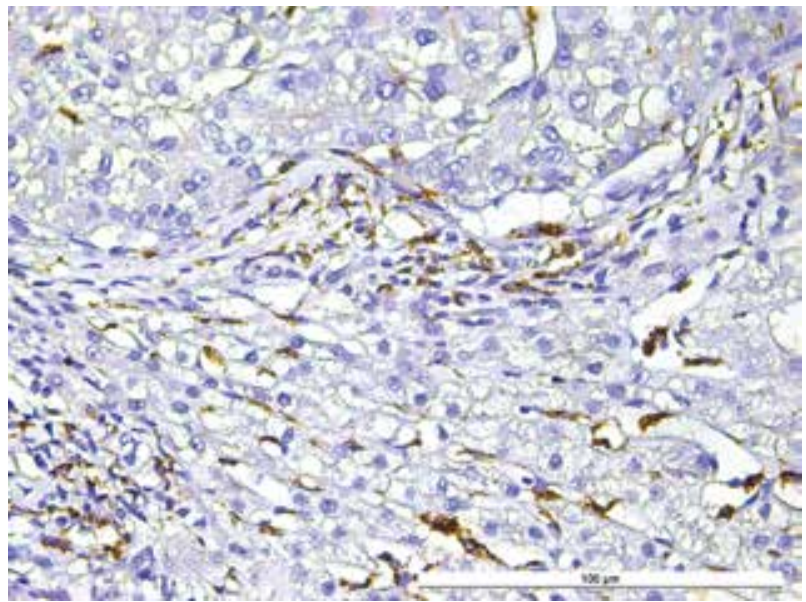

Figure 2. IHC detection of macrophages at the interface between $\mathrm{HCC}$ and normal liver parenchyma. Objective $\times 40$

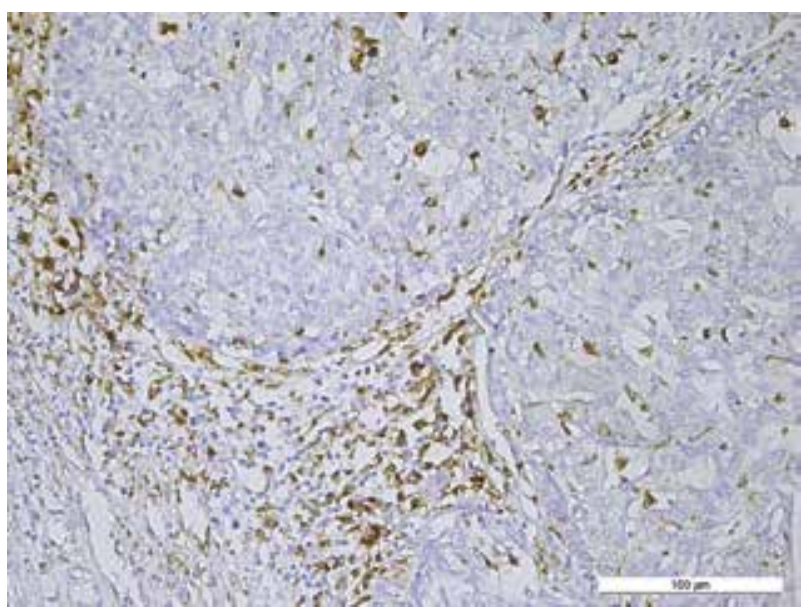

Figure 3. Distribution pattern of macrophages in HM: numerous at the border of the cancer proliferation, very few dispersed within tumor. IHC. Objective $\times 20$

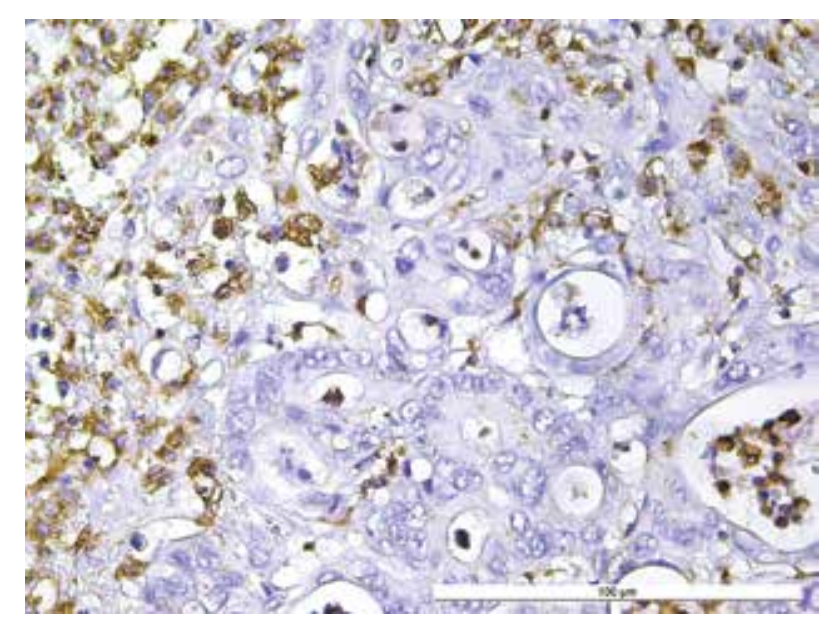

Figure 4. Macrophages disposed at the periphery of the metastatic nodule penetrating through the metastasized glands (colonic pseudocrypt). IHC. Objective $\times 40$ 
Table 3. Patients' clinical data and numbers of macrophages in Group 1 - HCC

\begin{tabular}{|c|c|c|c|c|c|c|c|c|c|}
\hline \multirow{3}{*}{$\begin{array}{l}\text { Case } \\
\text { number }\end{array}$} & \multicolumn{3}{|c|}{ Clinical variables } & \multicolumn{3}{|c|}{ Intratumoral macrophages } & \multicolumn{3}{|c|}{ Peritumoral macrophages } \\
\hline & \multirow[t]{2}{*}{ Age } & \multirow[t]{2}{*}{$\begin{array}{c}\text { Tumor } \\
\text { stage }\end{array}$} & \multirow[t]{2}{*}{$\begin{array}{l}\text { Histological } \\
\text { stage }\end{array}$} & \multirow[t]{2}{*}{$\begin{array}{l}\text { Numerical profile } \\
\quad(\text { mean } \pm \text { SD })\end{array}$} & \multicolumn{2}{|c|}{$\begin{array}{c}\text { TAMs } \\
\text { expression }\end{array}$} & \multirow[t]{2}{*}{$\begin{array}{l}\text { Numerical profile } \\
\quad(\text { mean } \pm \text { SD })\end{array}$} & \multicolumn{2}{|c|}{$\begin{array}{c}\text { TAMs } \\
\text { expression }\end{array}$} \\
\hline & & & & & $\mathbf{T 1}^{*}$ & $\mathbf{T} 2^{* *}$ & & $\mathbf{T 1}^{\&}$ & $T_{2}^{\text {\&\& }}$ \\
\hline 1 & 61 & pT3Nx & G1 & $118.30 \pm 23.22$ & $\sqrt{3}$ & $\uparrow$ & $303.90 \pm 132.33$ & 仓 & $\uparrow$ \\
\hline 2 & 63 & pT2Nx & G1 & $184.9 \pm 73.40$ & $\hat{\imath}$ & $\uparrow$ & $317.80 \pm 124.70$ & 仓े & 个 \\
\hline 3 & 68 & pT3Nx & G3 & $87.7 \pm 44.71$ & $\sqrt{n}$ & $\uparrow$ & $50.20 \pm 28.61$ & $\sqrt{3}$ & $\downarrow$ \\
\hline 4 & 54 & pT1Nx & G1 & $46.7 \pm 29.90$ & $\sqrt{n}$ & $\uparrow$ & $172.00 \pm 54.77$ & $\hat{\imath}$ & $\uparrow$ \\
\hline 5 & 48 & pT3Nx & G2 & $337.7 \pm 50.78$ & $\hat{\imath}$ & $\uparrow$ & $344.80 \pm 78.10$ & $\hat{\imath}$ & $\uparrow$ \\
\hline 6 & 59 & pT3Nx & G2 & $137.1 \pm 68.82$ & $\sqrt{3}$ & $\uparrow$ & $289.20 \pm 189.21$ & $\hat{\imath}$ & $\uparrow$ \\
\hline 7 & 57 & pT3Nx & G2 & $209.9 \pm 81.09$ & $\hat{v}$ & $\uparrow$ & $59.30 \pm 28.76$ & ת & $\downarrow$ \\
\hline 8 & 72 & pT1Nx & G1 & $3.9 \pm 2.46$ & ת & $\downarrow$ & $32.00 \pm 15.86$ & ת & $\downarrow$ \\
\hline 9 & 57 & pT3Nx & G3 & $167.10 \pm 98.92$ & 仓े & $\uparrow$ & $227.70 \pm 92.23$ & 仓े & $\uparrow$ \\
\hline 10 & 58 & pT2Nx & G3 & $68.00 \pm 25.15$ & $\sqrt{n}$ & $\uparrow$ & $272.60 \pm 87.18$ & $\hat{\imath}$ & $\uparrow$ \\
\hline 11 & 58 & pT2Nx & G3 & $105.30 \pm 97.32$ & $\sqrt{3}$ & $\uparrow$ & $156.10 \pm 118.65$ & 仓े & $\uparrow$ \\
\hline 12 & 74 & pT3Nx & G2 & $116.90 \pm 50.35$ & $\sqrt{n}$ & 个 & $248.30 \pm 79.78$ & $\hat{\imath}$ & 个 \\
\hline 13 & 51 & pT3Nx & $\mathrm{G} 2$ & $50.90 \pm 25.28$ & $\sqrt{3}$ & $\uparrow$ & $170.90 \pm 61.13$ & 仓 & $\uparrow$ \\
\hline 14 & 83 & pT2Nx & $\mathrm{G} 2$ & $85.10 \pm 50.34$ & $\sqrt{2}$ & $\uparrow$ & $376.50 \pm 106.96$ & 仓े & $\uparrow$ \\
\hline 15 & 65 & pT2Nx & G2 & $127.00 \pm 57.42$ & ת & $\uparrow$ & 0 & ת & $\downarrow$ \\
\hline 16 & 74 & pT2Nx & G1 & 0 & $\sqrt{3}$ & $\downarrow$ & $253.40 \pm 169.43$ & 仓े & $\uparrow$ \\
\hline 17 & 67 & pT2Nx & G3 & $418.60 \pm 137.75$ & $\hat{\imath}$ & $\uparrow$ & $578.50 \pm 126.59$ & 仓े & $\uparrow$ \\
\hline 18 & 74 & pT2Nx & G1 & $7.50 \pm 7.97$ & ת & $\downarrow$ & $76.20 \pm 49.08$ & ת & $\uparrow$ \\
\hline 19 & 70 & pT3Nx & $\mathrm{G} 2$ & $74.70 \pm 25.56$ & $\sqrt{3}$ & $\uparrow$ & $318.40 \pm 90.32$ & 仓 & $\uparrow$ \\
\hline 20 & 68 & pT3Nx & $\mathrm{G} 2$ & $43.80 \pm 12.56$ & $\sqrt{n}$ & $\uparrow$ & $58.00 \pm 30.38$ & ת & $\downarrow$ \\
\hline 21 & 64 & pT1Nx & G1 & $89.40 \pm 16.22$ & $\sqrt{n}$ & $\uparrow$ & $134.00 \pm 57.71$ & ת & $\uparrow$ \\
\hline 22 & 72 & pT1Nx & G1 & $63.20 \pm 33.15$ & $\sqrt{n}$ & $\uparrow$ & $195.10 \pm 108.28$ & $\hat{\imath}$ & $\uparrow$ \\
\hline 23 & 56 & pT3Nx & G2 & $91.70 \pm 11.84$ & $\sqrt{2}$ & $\uparrow$ & $247.70 \pm 105.86$ & 仓े & 个 \\
\hline 24 & 75 & pT3Nx & $\mathrm{G} 2$ & $89.40 \pm 23.15$ & $\sqrt{2}$ & $\uparrow$ & $52.10 \pm 15.96$ & ת & $\downarrow$ \\
\hline 25 & 60 & pT3Nx & $\mathrm{G} 2$ & $61.10 \pm 20.61$ & $\sqrt{3}$ & $\uparrow$ & $329.00 \pm 221.33$ & 仓े & $\uparrow$ \\
\hline 26 & 57 & pT2Nx & G3 & $23.10 \pm 13.21$ & ת & $\downarrow$ & $226.70 \pm 103.65$ & $\hat{\varphi}$ & 个 \\
\hline 27 & 75 & pT2Nx & G1 & $72.70 \pm 23.36$ & ת & $\uparrow$ & $613.20 \pm 123.69$ & 仓े & $\uparrow$ \\
\hline 28 & 58 & pT2Nx & $\mathrm{G} 2$ & 0 & $\sqrt{3}$ & $\downarrow$ & $281.50 \pm 136.75$ & 仓े & $\uparrow$ \\
\hline 29 & 58 & pT1Nx & G1 & $133.50 \pm 49.91$ & $\sqrt{3}$ & 个 & $117.20 \pm 50.83$ & ת & $\uparrow$ \\
\hline 30 & 71 & p2NxM1\# & G3 & $112.60 \pm 64.33$ & ת & 个 & $358.60 \pm 78.14$ & 仓े & $\uparrow$ \\
\hline 31 & 69 & pT3Nx & G1 & $121.30 \pm 28.14$ & $\sqrt{3}$ & $\uparrow$ & $248.60 \pm 62.96$ & 仓े & $\uparrow$ \\
\hline 32 & 53 & pT2Nx & G2 & $71.70 \pm 38.12$ & ת & 个 & $484.70 \pm 181.19$ & 仓े & $\uparrow$ \\
\hline 33 & 65 & pT3Nx & G1 & $7.90 \pm 4.40$ & $\sqrt{3}$ & $\downarrow$ & $148.20 \pm 81.24$ & 仓 & $\uparrow$ \\
\hline 34 & 23 & pT3Nx & G1 & $75.00 \pm 38.97$ & $\sqrt{n}$ & $\uparrow$ & $83.60 \pm 53.57$ & ת & $\uparrow$ \\
\hline 35 & 67 & pT2Nx & $\mathrm{G} 2$ & $98.20 \pm 65.55$ & $\sqrt{2}$ & $\uparrow$ & $151.30 \pm 75.47$ & 仓े & $\uparrow$ \\
\hline \multicolumn{4}{|c|}{ Mean \pm SEM } & $100.05 \pm 14.62$ & \multicolumn{5}{|c|}{$227.9 \pm 24.81$} \\
\hline \multicolumn{4}{|l|}{$\begin{array}{l}\text { Median, } \\
\text { range }\end{array}$} & $\begin{array}{c}87.70, \\
0-418.60\end{array}$ & \multicolumn{5}{|c|}{$\begin{array}{c}227.7 \\
0-613.2\end{array}$} \\
\hline \multicolumn{5}{|l|}{$P$} & \multicolumn{3}{|c|}{$<0.0001$} & & \\
\hline
\end{tabular}

*IT Threshold $1=145.36$; Subgroup $1=\Re ;$ Subgroup $2=\hat{\text { 讠 }}$

**IT Threshold $2=33.33$; Subgroup $1=\boldsymbol{\downarrow}$; Subgroup $2=\uparrow$

\&PT Threshold $1=144.16$; Subgroup $1=\Omega ;$ Subgroup $2=$ 仓

\&\&PT Threshold $2=75.96$; Subgroup $1=\boldsymbol{\downarrow}$; Subgroup $2=\uparrow$

\#HCC metastases were diagnosed in bone 
Table 4. Patients' clinical data and numbers of macrophages in Group $2-\mathrm{HM}$

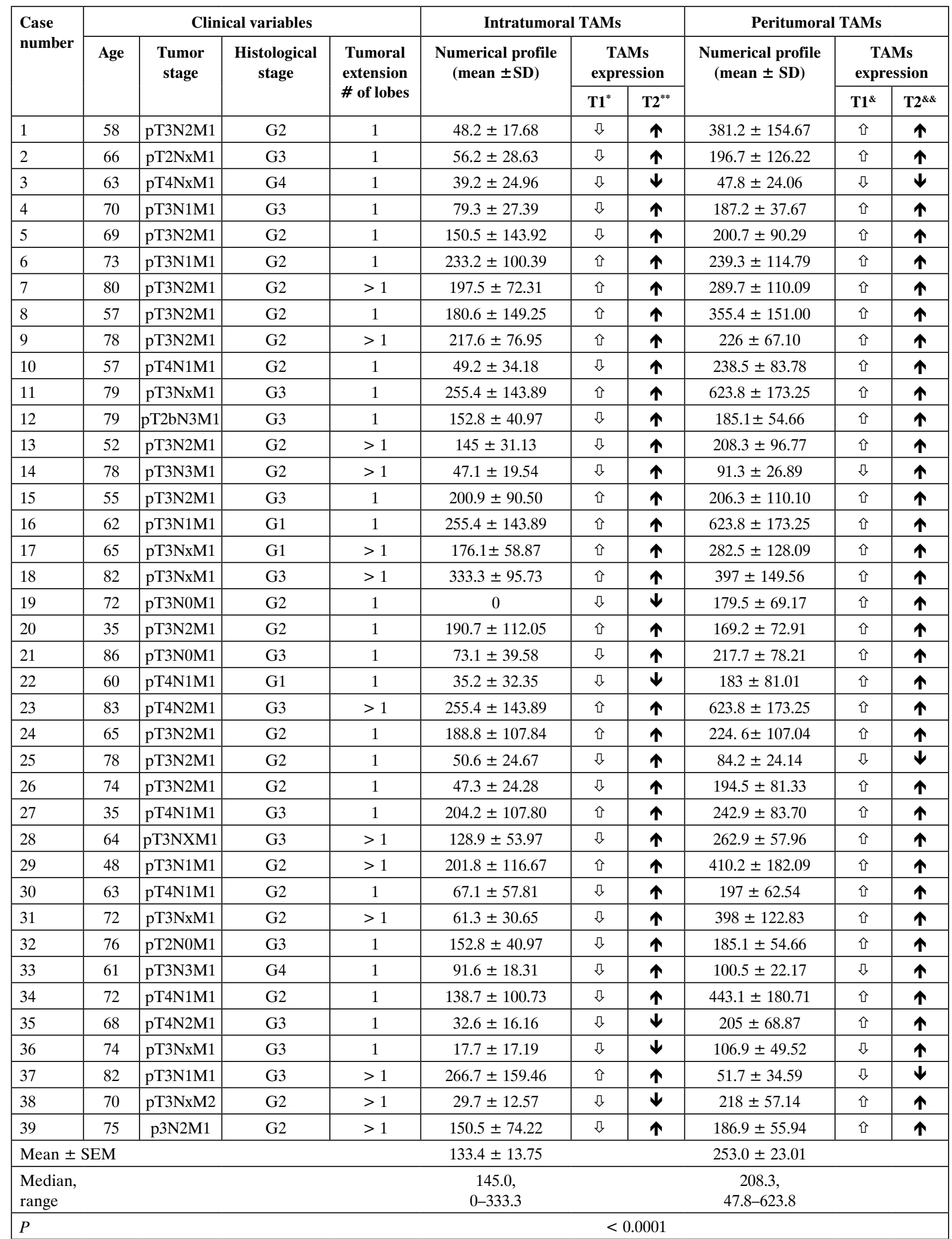

*IT Threshold $1=162.29 ;$ Subgroup $1=\sqrt{ } ;$ Subgroup $2=$ 仓

**IT Threshold $2=44.46$; Subgroup $1=\boldsymbol{\downarrow}$; Subgroup $2=\uparrow$

\&PT Threshold $1=163.00 ;$ Subgroup $1=\sqrt{ } ;$ Subgroup $2=$ 仓

\&\&T Threshold $2=84.33$; Subgroup $1=\boldsymbol{\downarrow}$; Subgroup $2=\uparrow$ 


\section{Quantitative evaluation}

Table 3 and 4 present the numerical values of the macrophages quantification as well as clinical variables, in each individual case of both HCC and HM groups. Since TAMs scores or intratumoral subgroups did not pass D'Agostino normality tests, we used non-parametric tests for further statistical analyzes.

\section{TAMs in hepatocellular carcinoma group}

Morphometric analysis revealed more than 2-fold lower number of TAMs in IT vs. PT compartment in HCC tumors $(P<0.0001$; Table 3, Figure 5A). Threshold 1 (T1) values were calculated using Cox $(-\log )$ test as 145.36 for IT and 144.16 for PT macrophages, whereas Threshold 2 (T2) values were counted as 1/3 of mean values for subgroups as 33.33 for IT and 75.92 for PT groups (Table 3).

The analysis of IT macrophages by using threshold 1 revealed the presence of $30 / 35(86 \%)$ cases with lower score than $\mathrm{T} 1$. If $\mathrm{T} 2$ threshold was applied we observed a different pattern, since 6/35 (17\%) cases showed even lower TAMs presence than $\mathrm{T} 2$, therefore $\mathrm{T} 1$ and $\mathrm{T} 2$ classifications cannot be compared $(P<0.0001$, Fisher's test $)$.

The analysis of IT macrophages in peritumoral territory of HCC revealed that $10 / 35(28.5 \%)$ and $6 / 35$ $(17 \%)$ cases showed lower number of TAMs when T1 or T2 were utilized, respectively. In this approach (PT in HCC), both methods of threshold determination can be compared ( $P=0.39$; Fisher's test).

We found no statistical associations between TAMs scores and clinical data of HCC cases (data not shown). We did not find any correlation between number of macrophages in intratumoral and peritumoral tissue (Figure 5B).

\section{TAMs in hepatic metastases}

Similarly to HCC, twice more TAMs were present in peritumoral than in intratumoral territories of $\mathrm{HM}$ patients $(P<0.0001$, Table 4; Figure 5A). T1 and T2 for IT area were calculated as 162.29 and 44.46, respectively. Within IT tissue we observed decreased number of TAMs for $24 / 39(61.5 \%)$ or $6 / 39(15.3 \%)$ when T1 or T2 values were applied, respectively. For peritumoral HM cases, with T1 and T2 set for 163 and 84.33 , respectively, we found decreased number of TAMs in $6 / 39(15.3 \%)$ if T1 criterion was used or $3 / 39(7.7 \%)$ if $\mathrm{T} 2$ was applied.

For PT in HM, T1 and T2 can be compared $(P=$ 0.48), whereas for IT in HM there was no agreement between T1 and T2 classification methods ( $P=0.001$, Fisher's $2 \times 2$ test).
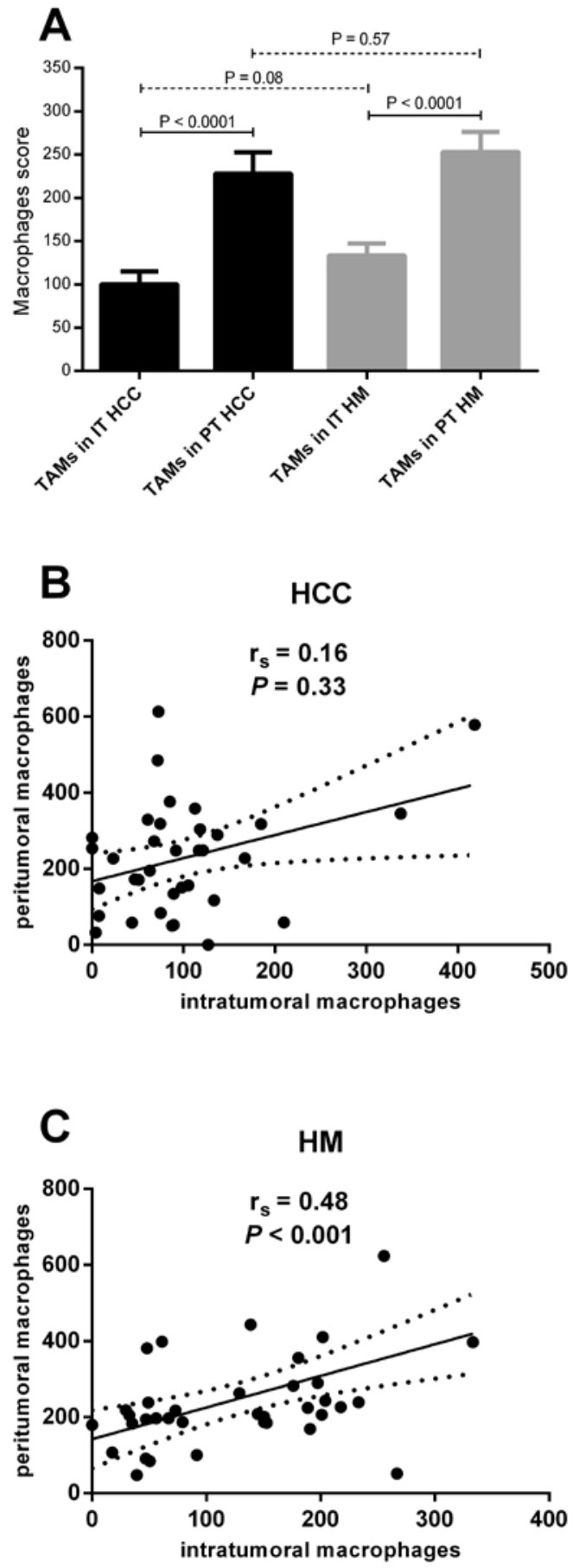

Figure 5. Comparison between macrophages numbers classified by cancer origin and liver territory. A. Plot of mean $( \pm$ SEM) number of TAMs and the results of Mann-Whitney $U$ test. $\mathbf{B}$ and $\mathbf{C}$. correlation plots of TAMs number in matched HCC and HM cases with results of Spearman's test. HCC - hepatocellular carcinoma, HM - hepatic metastasis, IT — intratumoral, PT — peritumoral territory

Although we did not find any statistical associations between TAMs scores in HM and clinical data, we noticed a positive correlation between IT and PT sco$\operatorname{res}\left(\mathrm{r}_{\mathrm{s}}=0.48, P<0.0001\right.$; Spearman's test, Figure 5C). 
Table 5. HCC and HM clinical data in relation to decreased number of macrophages in intratumoral and peritumoral territories

\begin{tabular}{|c|c|c|c|c|}
\hline \multirow[t]{2}{*}{ Clinicopathological factor } & \multicolumn{2}{|c|}{ Intratumoral territory } & \multicolumn{2}{|c|}{ Peritumoral territory } \\
\hline & $\begin{array}{c}\text { T1 } \\
\text { תvs. 仑 }\end{array}$ & $\begin{array}{c}\text { T2 } \\
\downarrow_{v s .} \uparrow\end{array}$ & $\begin{array}{c}\text { T1 } \\
\Omega v s . \text { ثิ }\end{array}$ & $\begin{array}{c}\text { T2 } \\
\downarrow v s . \uparrow\end{array}$ \\
\hline \multicolumn{5}{|l|}{ HCC } \\
\hline $\begin{array}{l}\text { Age } \\
\quad<63 \\
\geq 63\end{array}$ & $\begin{array}{l}13 \text { vs. } 3 \\
P=0.6 \\
17 \text { vs. } 2\end{array}$ & $\begin{array}{c}2 \text { vs. } 14 \\
P=0.66 \\
4 \text { vs. } 15\end{array}$ & $\begin{array}{c}3 \text { vs. } 13 \\
P=0.28 \\
7 \text { vs. } 12\end{array}$ & $\begin{array}{c}1 \text { vs. } 15 \\
P=0.18 \\
5 \text { vs. } 14\end{array}$ \\
\hline $\begin{array}{l}\text { Tumor stage } \\
\text { I-II } \\
\text { III-IV }\end{array}$ & $\begin{array}{c}16 \text { vs. } 2 \\
P=0.65 \\
14 \text { vs. } 3\end{array}$ & $\begin{array}{c}5 \text { vs. } 13 \\
P=0.17 \\
1 \text { vs. } 16\end{array}$ & $\begin{array}{c}5 \text { vs. } 13 \\
P=1 \\
5 \text { vs. } 12 \\
\end{array}$ & $\begin{array}{l}2 \text { vs. } 16 \\
P=0.4 \\
4 \text { vs. } 13\end{array}$ \\
\hline $\begin{array}{l}\text { Histological grade } \\
\text { G1-G2 } \\
\text { G3 }\end{array}$ & $\begin{array}{c}25 \text { vs. } 3 \\
P=0.25 \\
5 \text { vs. } 2\end{array}$ & $\begin{array}{l}5 v s .23 \\
P=1 \\
1 \text { vs. } 6 \\
\end{array}$ & $\begin{array}{c}9 \text { vs. } 19 \\
P=0.64 \\
1 \text { vs. } 6\end{array}$ & $\begin{array}{l}5 v s .23 \\
P=1 \\
1 \text { vs. } 6\end{array}$ \\
\hline \multicolumn{5}{|l|}{ HM } \\
\hline $\begin{array}{l}\text { Age } \\
\leq 68 \\
>68\end{array}$ & $\begin{array}{l}10 \text { vs. } 8 \\
P=0.2 \\
21 \text { vs. } 7\end{array}$ & $\begin{array}{c}3 \text { vs. } 15 \\
P=0.65 \\
3 \text { vs. } 25\end{array}$ & $\begin{array}{c}2 \text { vs. } 16 \\
P=0.66 \\
4 \text { vs. } 17\end{array}$ & $\begin{array}{c}1 \text { vs. } 17 \\
P=1 \\
2 \text { vs. } 19 \\
\end{array}$ \\
\hline $\begin{array}{l}\text { Histological grade } \\
\text { G1-G2 } \\
\text { G3-G4 }\end{array}$ & $\begin{array}{l}14 \text { vs. } 9 \\
P=0.2 \\
6 \text { vs. } 10\end{array}$ & $\begin{array}{c}3 \text { vs. } 20 \\
P=0.67 \\
3 \text { vs. } 13\end{array}$ & $\begin{array}{l}2 \text { vs. } 21 \\
P=0.2 \\
4 \text { vs. } 12\end{array}$ & $\begin{array}{c}1 \text { vs. } 22 \\
P=0.55 \\
2 \text { vs. } 14\end{array}$ \\
\hline $\begin{array}{l}\text { Tumor extension } \\
\text { One lobe } \\
\text { Many lobes }\end{array}$ & $\begin{array}{c}18 \text { vs. } 8 \\
P=0.18 \\
6 \text { vs. } 7\end{array}$ & $\begin{array}{c}5 \text { vs. } 21 \\
P=0.64 \\
1 \text { vs. } 12\end{array}$ & $\begin{array}{c}4 \text { vs. } 22 \\
P=1 \\
2 \text { vs. } 11\end{array}$ & $\begin{array}{c}2 v s .24 \\
P=1 \\
1 \text { vs. } 12\end{array}$ \\
\hline
\end{tabular}

Furthermore, there was no difference between number of macrophages in HM or HCC when IT or PT territories were compared (Figure 5A).

In the explorative analysis of patients with observed lower number of TAMs, either based on T1 or T2 scoring method, we did not observe any association between occurrence of low/high numbers of TAMs and age, TNM, histological G grade in HCC or age, histological $\mathrm{G}$ grade and number of involved lobes in HM (Table 5).

\section{Analysis of prognostic factors}

Univariate and multivariate Cox regression analysis was used in order to find the factors which can influence patients' survival or death. The summary analysis of all variables was presented in Table 6 . In HCC group we observed that increasing tumor stage is associated with poorer prognosis $(P=0.013, \mathrm{HR}$ 2.16 ), which is related to $\times 13.6$ increase of chance of death for patients with stages III-IV in comparison to $\mathrm{HCC}$ cases with diagnosed $\mathrm{HCC}$ at stages I-II. In liver metastasis we observed that increasing histological grades of cancer cells (G1-G2 vs. G3-G4) as well as increasing number of metastatic lobes $(>1)$ are the factors of poorer prognosis $(P=0.004$ for both factors). We further included both factors in Cox multivariate analysis and retained their significance ( $P=0.021$ and $\mathrm{P}=0.045$ respectively). For the prognostic significance of TAMs scores, we did not find relations in any of analyzed groups, no matter which threshold was applied.

\section{Survival analysis}

The possible influence of all clinical factors as well as TAMs scores based either on T1 or T2 classifications were assessed separately for IT and PT areas. We found that HCC cases with PT TAMs scores lower than $1 / 3$ mean value (T2) were characterized by a shorter survival rate that cases with higher PT TAMs values (median survival 1 vs. 8 months, 3 years survival $0 \%$ vs. 38\%; Figure 6A). Other survival relations to TAMs were not significant. However, certain clinical factors had significant influence on HCC and HM survival rates: Stages I-II vs. stages IIIA-IV (median survival 28 vs. 3.5 months, 3 years survival $42 \%$ vs. $21 \%$, Figure 6B). 
Table 6. Univariate and multivariate Cox regression analysis of hepatocellular carcinoma and hepatic metastases cases

\begin{tabular}{|c|c|c|c|c|}
\hline \multirow[t]{2}{*}{ Parameters } & \multicolumn{2}{|c|}{ Univariate analysis } & \multicolumn{2}{|c|}{ Multivariate analysis } \\
\hline & $P$ value & HR $(95 \% \mathrm{CI})$ & $P$ value & HR $(95 \%$ CI $)$ \\
\hline \multicolumn{5}{|l|}{ HCC } \\
\hline Age & 0.836 & $1.004(0.961-1.049)$ & & \\
\hline $\begin{array}{l}\text { Histological grade } \\
\text { G1-G2 vs. G3 }\end{array}$ & 0.316 & $1.382(0.733-2.603)$ & & \\
\hline $\begin{array}{l}\text { Tumor stage } \\
\leq \text { II } v s . \geq \text { III }\end{array}$ & 0.013 & $2.614(1.209-5.410)$ & & \\
\hline $\begin{array}{l}\text { Intratumoral TAMs } \\
\text { T1 仓v vs. } \sqrt{ } \\
\text { T2 } \uparrow v s . \downarrow\end{array}$ & $\begin{array}{l}0.666 \\
0.968\end{array}$ & $\begin{array}{l}1.269(0.429-3.751) \\
1.022(0.347-3.009)\end{array}$ & & \\
\hline $\begin{array}{l}\text { Peritumoral TAMs } \\
\text { T1 仓vs. 』 } \\
\text { T2 } \uparrow v s . \downarrow\end{array}$ & $\begin{array}{l}0.432 \\
0.062\end{array}$ & $\begin{array}{l}1.434(0.582-3.532) \\
2.457(0.954-6.326)\end{array}$ & & \\
\hline \multicolumn{5}{|l|}{ HM } \\
\hline $\begin{array}{l}\text { Age } \\
\text { Histological grade } \\
\text { G1-G2 vs. G3-G4 }\end{array}$ & $\begin{array}{l}0.187 \\
\mathbf{0 . 0 0 4}\end{array}$ & $\begin{array}{l}1.025(0.987-1.064) \\
\mathbf{1 . 7 0 1}(\mathbf{1 . 0 2 4 - 2 . 8 2 7})\end{array}$ & 0.021 & $0.395(0.179-0.870)$ \\
\hline Tumoral extension one lobe $v s$. multilobe & 0.004 & $0.498(0.225-1.101)$ & 0.045 & $0.438(0.195-0.984)$ \\
\hline $\begin{array}{l}\text { Intratumoral TAMs } \\
\text { T1 îvs. } \Omega \\
\text { T2 } \uparrow v s . \downarrow\end{array}$ & $\begin{array}{l}0.966 \\
0.973\end{array}$ & $\begin{array}{c}0.983(0.444-2.175) \\
1.018\end{array}$ & & \\
\hline $\begin{array}{l}\text { Peritumoral TAMs } \\
\text { T1 仓ैvs. } \\
\text { T2 } \uparrow v s . \downarrow\end{array}$ & $\begin{array}{l}0.161 \\
0.914\end{array}$ & $\begin{array}{l}2.028 \\
1.082\end{array}$ & & \\
\hline
\end{tabular}

Cox analysis of $\hat{v} v s . \sqrt{ }$ or $\uparrow v s . \boldsymbol{\downarrow}$ means that the HR and P values relate to occurrence of decrease number of TAMs

For HM we observed the poorer survival if many lobes were involved: one vs. multilobe involvement (median survival 31 vs. 5 months, 3 years survival $29 \%$ vs. $15 \%$, Figure 6C) or if more aggressive tumor cells were found in tumor biopsies: G1-G2 vs. G3-G4 (median survival 34 vs. 3.5 months, 3 years survival $43 \%$ vs. $6 \%$, Figure $6 \mathrm{D}$ ). We also noted that patients with HCC had worse survival outcome than HM cases $(P=0.0501$, Figure 6E).

\section{Discussion}

Recent data presented the modulatory role of hepatic microenvironment both in initiation and progression of the primary carcinogenic process as well as in establishment of optimal conditions for metastatic colonization and development [5, 12, 21, 26]. A necessary condition for HCC initiation and development seems to be the presence of activated inflammatory cells in the liver, through their action on signaling pathways, which results in enhanced cellular turnover and acquisition of critical modifications for the malignant transformation [27, 28].
Studies on inflammatory cells in cancer development focused mainly on the population of tumor-infiltrating lymphocytes (TILs), widely reported in CRC [29-35], however, within liver microenvironment the largest physiological population of immune cells is represented by macrophages, namely Kupfer cells. Our study focused on the parallel analysis of hepatic TAMs in two different tumor pathologies, HCC and $\mathrm{HM}$ by evaluating the presence of macrophages in intra- and peritumoral territories, followed by TAMs quantification in respective territories.

Regarding the method of counting TAMs and due to the fact that no accurate correlation between number of macrophages and patients' survival could be found in literature, we established two threshold values. T1 was calculated with the use of widely used Cox method, however, the setting of a second threshold, T2, permitted a convenient mathematical manipulation and had a heuristic motivation (based on the large panel of numerical results). Choice of $\mathrm{T} 2$ intended to balance the following two issues: 1 ) the use of the mean value of the number of macrophages does not ensure a reliable classification of tissues with decreased number 

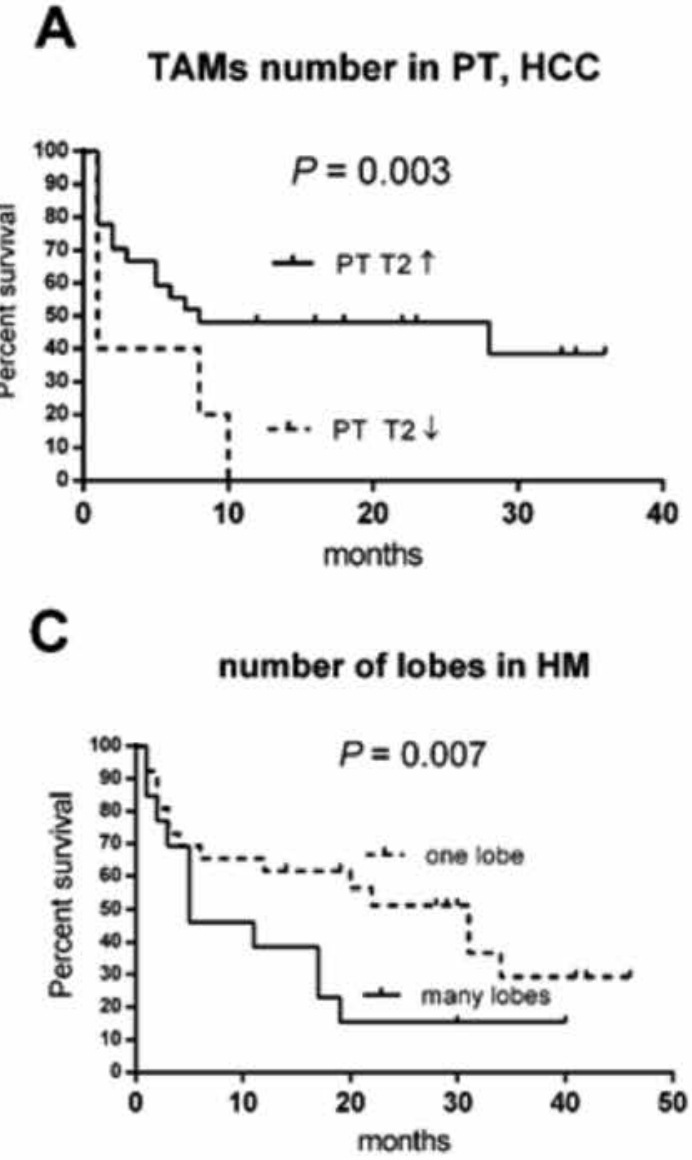

E

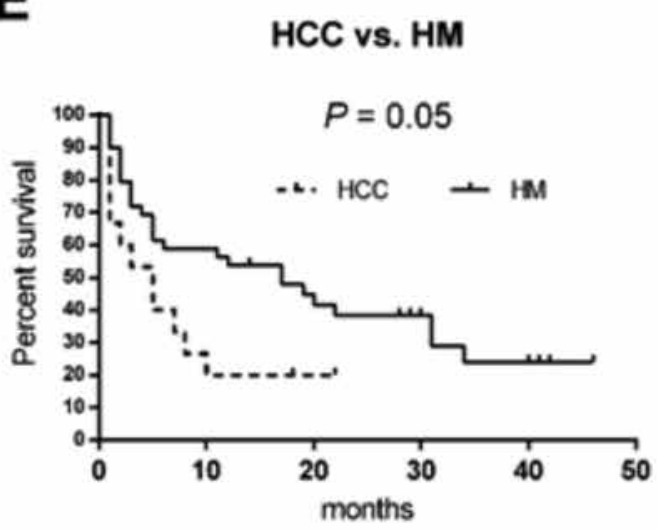

B

HCC stages

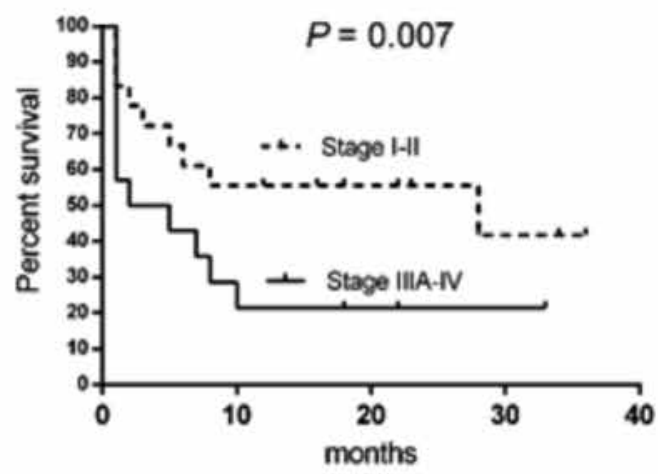

D histological grade in HM

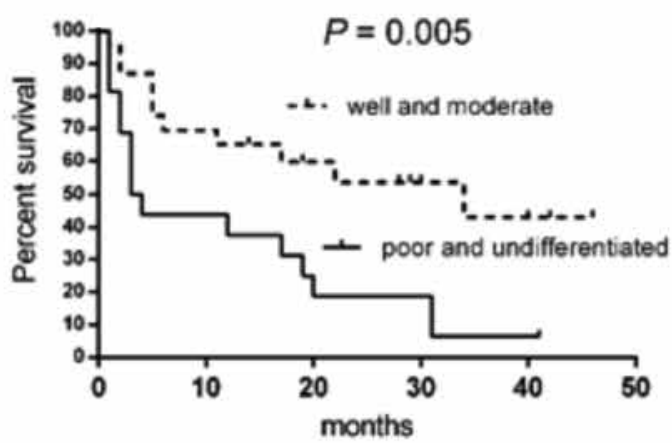

Figure 6. Results of Kaplan-Meier survival analyzes of chosen $(P<0.05)$ factors. A. HCC cases classified by higher $(\boldsymbol{\uparrow})$ and lower $(\boldsymbol{\downarrow})$ number of peritumoral macrophages. B. HCC cases divided by pTNM stages according to Benson et al. [43]. C. and D. Relates to survival analyses of HM cases divided by tumoral extension and histological grade of differentiation. E. Plot relates to survival outcome in relation to tumor disease type. $P$ values according to Mantel-Cox log-rank test

of TAMs, 2) a proper evaluation of the association of macrophages' number on survival requires a reasonable value above the zero. We however found that our method for T2 assignment could not be applied for TAMs in IT territories (both in HCC and HM), which was probably associated with lack of normal distribution of the macrophages' number in the intratumoral sections.
Our finding of decreased presence of intratumoral TAMs in comparison to peritumoral territories both in HCC and HM are in accordance with the relatively few studies of TAMs in HCC $[13,15,16]$ and HM $[18,20,36]$, confirming their predominant presence in peritumoral area and in the vicinity of invasion front. Moreover, our results support the 
hypothesis of decreased macrophage infiltration or the inhibition of their function which resulted in tumor growth and metastasis [28]. Surprisingly, despite the diversity of the origin of primary and secondary liver tumors, lack of differences in TAMs numbers between primary $v s$. secondary tumors in respective territories could have been caused by specific characteristics of hepatic microenvironment in recruiting and inducing the phenotype of tumor-associated macrophages either in HCC or HM [28]. Consequently, the macrophage distribution pattern suggests that the anti-tumoral defense mechanism follows a similar algorithm in the liver, without any correlation to the tumor origin or to the primary or secondary tumor status $[1,11]$.

Our observation of positive correlation of TAMs numbers in IT and PT tumor sections of HM patients could be probably associated with the impact of malignant cells on hepatic macrophages. It was reported in HM that highly aggressive Dukes C and D malignant cells of CRC-origin can trigger apoptotic death of TAMs and monocytes by the secretion of high mobility group box 1 (HMGB1) protein [38]. On the other hand, malignant cells can attract TAMs by secretion of carcinoembryonic antigen (CEA) followed by the release of growth factors by enticed macrophages [21].

The presented study focused on the selection of prognostic factors for $\mathrm{HCC}$ and $\mathrm{HM}$ patients. Our results confirmed the usefulness of AJCC/UICC pTNM division for the prediction of the survival rate and hazard ratio of HCC patients [39, 40]. The influence of poorly/undifferentiated cells and involvement of many liver lobes in secondary cancer on the poorer outcome of HM patients has been previously reported [41].

Although TAMs presence in tumor microenvironment was observed in many types of cancer [37], the value of estimating number of macrophages in tumor infiltrates as a prognostic factor in HCC is still uncertain since some studies found TAMs number as a negative $[13,14]$, while other - as a positive prognosis factor $[15,16]$. Our results suggest that the low number of PT macrophages $(P=0.07)$ could be assumed as a negative prognostic factor for $\mathrm{HCC}$, which was further demonstrated by Kaplan-Meier analysis, however, we found no association with other clinical factors. Although this preliminary result should be confirmed by larger independent analyses, we wanted to emphasize such interesting observation.

One of the limitations of our study is related to the division of mature macrophages into two subpopulations: M1 and M2. M1 macrophages activated mainly by bacterial lipopolysaccharides and immune stimuli such as interferon- $\gamma($ IFN- $\gamma$ ) have anti-tumor role due to the elimination of tumor cells, antigen presentation to T cells, and synthesis of numerous proinflammatory cytokines [6-8]. M2 macrophages differentiate following the contact with Th2 cells or after stimulation in the tumor microenvironment by cytokines (e.g. IL-4, IL-10, IL-13) and growth factors such as TGF- $\beta 1$ [42]. Since we performed only basic identification for macrophages, future studies should assess the participation of M1 and M2 subpopulation of macrophages in HCC and HM. In this report we were not able to simultaneously detect CD68+, CD80+ and CD163+ cells in histological sections but the use of flow-cytometry to study isolated TAMs may provide valuable data.

In summary, our results lead to the following two conclusions. (i) Low number of intratumoral TAMs in comparison to peritumoral territories both in HCC and $\mathrm{HM}$ may be related to negative influence of cancer cells. (ii) Increased TAMs number in peritumoral territory of hepatocellular carcinoma is associated with better prognosis.

\section{Acknowledgement}

The first author acknowledges the support of the POSDRU/88/1.5/S/78702 project, financed by the European Social Fund and the Romanian Government.

\section{References}

1. Yang JD, Nakamura I, Roberts LR. The tumor microenvironment in hepatocellular carcinoma: current status and therapeutic targets. Semin Cancer Biol. 2011;21:35-43.

2. Hernandez-Gea V, Toffanin S, Friedman SL, Llovet JM. Role of the microenvironment in the pathogenesis and treatment of hepatocellular carcinoma. Gastroenterology. 2013;144:512-527.

3. Knolle PA, Gerken G. Local control of the immune response in the liver. Immunol Rev. 2000;174:21-34.

4. Diehl L, Schurich A, Grochtmann R, Hegenbarth S, Chen L, Knolle PA. Tolerogenic maturation of liver sinusoidal endothelial cells promotes B7-homolog 1-dependent CD8+ T cell tolerance. Hepatology. 2008;47:296-305.

5. Solinas G, Germano G, Mantovani A, Allavena P. Tumor-associated macrophages (TAM) as major players of the cancer -related inflammation. J Leukoc Biol. 2009;86:1065-1073.

6. Mantovani A, Sozzani S, Locati M, Allavena P, Sica A. Macrophage polarization: tumor-associated macrophages as a paradigm for polarized M2 mononuclear phagocytes. Trends Immunol. 2002;23:549-555.

7. Mantovani A, Sica A. Macrophages, innate immunity and cancer: balance, tolerance, and diversity. Curr Opin Immunol. 2010;22:231-237.

8. Qian BZ, Pollard JW. Macrophage diversity enhances tumor progression and metastasis. Cell. 2010;141:39-51.

9. Mantovani A, Sica A, Allavena P, Garlanda C, Locati M. Tumor-associated macrophages and the related myeloid-derived suppressor cells as a paradigm of the diversity of macrophage activation. Hum Immunol. 2009;70:325-330.

10. Stout RD, Suttles J. Functional plasticity of macrophages: reversible adaptation to changing microenvironments. J Leukoc Biol. 2004;76:509-513. 
11. Quatromoni JG, Eruslanov E. Tumor-associated macrophages: function, phenotype, and link to prognosis in human lung cancer. Am J Transl Res. 2012;4:376-389.

12. Dent P. Janus-faced Kupffer cells in tumor metastasis. Cancer Biol Ther. 2013;14:875-876.

13. Ding $\mathrm{T}, \mathrm{Xu} \mathrm{J}$, Wang $\mathrm{F}$ et al. High tumor-infiltrating macrophage density predicts poor prognosis in patients with primary hepatocellular carcinoma after resection. Hum Pathol. 2009;40:381-389.

14. Capece D, Fischietti M, Verzella D et al. The inflammatory microenvironment in hepatocellular carcinoma: a pivotal role for tumor-associated macrophages. Biomed Res Int. 2013;2013:187-204.

15. Li YW, Qiu SJ, Fan J et al. Tumor-infiltrating macrophages can predict favorable prognosis in hepatocellular carcinoma after resection. J Cancer Res Clin Oncol. 2009;135:439-449.

16. Chew V, Tow $\mathrm{C}$, Teo $\mathrm{M}$ et al. Inflammatory tumour microenvironment is associated with superior survival in hepatocellular carcinoma patients. J Hepatol. 2010;52:370-379.

17. Miyagawa S, Miwa S, Soeda J, Kobayashi A, Kawasaki S. Morphometric analysis of liver macrophages in patients with colorectal liver metastasis. Clin Exp Metastasis. 2002;19:119-125.

18. Giusca SE, Zugun FE, Tarcoveanu E, Carasevici E, Amalinei C, Caruntu ID. Immunohistochemical study of colorectal cancer liver metastases: the immune/inflammatory infiltrate. Rom J Morphol Embryol. 2010;51:73-79.

19. Zhou Q, Peng RQ, Wu XJ et al. The density of macrophages in the invasive front is inversely correlated to liver metastasis in colon cancer. J Transl Med. 2010;8:13.

20. Keim S, Zoernig I, Spille A et al. Sequential metastases of colorectal cancer: Immunophenotypes and spatial distributions of infiltrating immune cells in relation to time and treatments. Oncoimmunology. 2012;1:593-599.

21. Paschos KA, Majeed AW, Bird NC. Natural history of hepatic metastases from colorectal cancer - pathobiological pathways with clinical significance. World J Gastroenterol. 2014;20:3719-3737.

22. Bayon LG, Izquierdo MA, Sirovich I, van Rooijen N, Beelen RH, Meijer S. Role of Kupffer cells in arresting circulating tumor cells and controlling metastatic growth in the liver. Hepatology. 1996;23:1224-1231.

23. Bilzer M, Roggel F, Gerbes AL. Role of Kupffer cells in host defense and liver disease. Liver Int. 2006;26:1175-1186.

24. Gorden DL, Fingleton B, Crawford HC, Jansen DE, Lepage M, Matrisian LM. Resident stromal cell-derived MMP-9 promotes the growth of colorectal metastases in the liver microenvironment. Int J Cancer. 2007;121:495-500.

25. Forssell J, Oberg A, Henriksson ML, Stenling R, Jung A, Palmqvist R. High macrophage infiltration along the tumor front correlates with improved survival in colon cancer. Clin Cancer Res. 2007;13:1472-1479.

26. Mantovani A, Germano G, Marchesi F, Locatelli M, Biswas SK. Cancer-promoting tumor-associated macrophages: new vistas and open questions. Eur J Immunol. 2011;41:2522-2525.
27. Budhu A, Wang XW. The role of cytokines in hepatocellular carcinoma. J Leukoc Biol. 2006;80:1197-1213.

28. Leonardi GC, Candido S, Cervello $\mathrm{M}$ et al. The tumor microenvironment in hepatocellular carcinoma (review). Int J Oncol. 2012;40:1733-1747.

29. Ropponen KM, Eskelinen MJ, Lipponen PK, Alhava E, Kosma VM. Prognostic value of tumour-infiltrating lymphocytes (TILs) in colorectal cancer. J Pathol. 1997;182:318-324.

30. Dolcetti R, Viel A, Doglioni C et al. High prevalence of activated intraepithelial cytotoxic $\mathrm{T}$ lymphocytes and increased neoplastic cell apoptosis in colorectal carcinomas with microsatellite instability. Am J Pathol. 1999;154:1805-1813.

31. Guidoboni M, Gafa R, Viel A et al. Microsatellite instability and high content of activated cytotoxic lymphocytes identify colon cancer patients with a favorable prognosis. Am J Pathol. 2001;159:297-304.

32. Dunn GP, Old LJ, Schreiber RD. The immunobiology of cancer immunosurveillance and immunoediting. Immunity. 2004;21:137-148.

33. Galon J, Costes A, Sanchez-Cabo F et al. Type, density, and location of immune cells within human colorectal tumors predict clinical outcome. Science. 2006;313:1960-1964.

34. Galon J, Fridman WH, Pages F. The adaptive immunologic microenvironment in colorectal cancer: a novel perspective. Cancer Res. 2007;67:1883-1886.

35. Ohtani H. Focus on TILs: prognostic significance of tumor infiltrating lymphocytes in human colorectal cancer. Cancer Immun. 2007;7:4.

36. Cui YL, Li HK, Zhou HY, Zhang T, Li Q. Correlations of tumor-associated macrophage subtypes with liver metastases of colorectal cancer. Asian Pac J Cancer Prev. 2013;14:1003-1007 .

37. Lin EY, Nguyen AV, Russell RG, Pollard JW. Colony-stimulating factor 1 promotes progression of mammary tumors to malignancy. J Exp Med. 2001;193:727-740.

38. Luo Y, Ohmori H, Fujii K et al. HMGB1 attenuates anti-metastatic defence of the liver in colorectal cancer. Eur J Cancer. 2010;46:791-799.

39. Kee KM, Wang JH, Lee CM et al. Validation of clinical AJCC/UICC TNM staging system for hepatocellular carcinoma: analysis of 5,613 cases from a medical center in southern Taiwan. Int J Cancer. 2007;120:2650-2655.

40. Yan T, Zhao JJ, Bi XY et al. Prognosis of hepatocellular carcinoma: a study of 832 cases. Zhonghua Zhong Liu Za Zhi. 2013;35:54-58.

41. Wyld L, Gutteridge E, Pinder SE et al. Prognostic factors for patients with hepatic metastases from breast cancer. $\mathrm{BrJ}$ Cancer. 2003;89:284-290.

42. Siveen KS, Kuttan G. Role of macrophages in tumour progression. Immunol Lett. 2009;123:97-102.

43. Benson AB 3rd, Abrams TA, Ben-Josef E et al. NCCN clinical practice guidelines in oncology: hepatobiliary cancers. $J$ Natl Compr Canc Netw. 2009;7:350-391.

Submitted: 4 January, 2014 Accepted after reviews: 13 June, 2014 